\title{
GLOBAL SOLUTION TO THE ALLEN-CAHN EQUATION WITH SINGULAR POTENTIALS AND DYNAMIC BOUNDARY CONDITIONS
}

\author{
Luca Calatroni \\ Cambridge Centre for Analysis, University of Cambridge \\ Wilberforce Road, CB3 0WA, Cambridge, United Kingdom \\ (lc524@cam.ac.uk) \\ Pierluigi Colli \\ Dipartimento di Matematica "F. Casorati", Università di Pavia \\ Via Ferrata 1, 27100, Pavia, Italy \\ (pierluigi.colli@unipv.it)
}

\begin{abstract}
We prove well-posedness results for the solution to an initial and boundaryvalue problem for an Allen-Cahn type equation describing the phenomenon of phase transitions for a material contained in a bounded and regular domain. The dynamic boundary conditions for the order parameter have been recently proposed by some physicists to account for interactions with the walls. We show our results using suitable regularizations of the nonlinearities of the problem and performing some a priori estimates which allow us to pass to the limit thanks to compactness and monotonicity arguments.
\end{abstract}

Key words: Allen-Cahn equation, dynamic boundary conditions, maximal monotone graphs, initial boundary value problem, existence and uniqueness results.

AMS Subject Classification: 35K55, 35K61, 80A22.

\section{Introduction}

The Allen-Cahn equation was originally introduced in [1] as a phenomenological model for anti-phase domain coarsening in a binary alloy. It has been subsequently applied to a wide range of other different problems such as the motion by mean curvature flows (cf., e.g., [12]) and the crystal growth (cf., e.g., [23]). In this paper we deal with a physical model which exploits this equation to describe the phenomenon of phase transitions (cf. [6]).

Let us consider a material occupying at any time $t \geq 0$ a bounded and connected domain $\Omega \subset \mathbb{R}^{d}, d=2,3$ with a smooth boundary $\Gamma:=\partial \Omega$. We suppose that the material can exhibit two different phases. The following semilinear parabolic partial 
differential equation proposed by Allen and Cahn in [1 models the time evolution of the order parameter $u$ in the isothermal case:

$$
\partial_{t} u-\Delta u+W^{\prime}(u)=0 \quad \text { in } \Omega \times(0,+\infty) .
$$

The nonlinearity $W$ is the potential associated with the phase configuration. In the literature (see, e.g., [11, 13, 20]) $W$ is frequently assumed to be a double-well potential, i.e. $W(u)=\frac{1}{4}\left(u^{2}-1\right)^{2}$. Usually, the values $u= \pm 1$ represent the pure states of the material, whereas the values inside the open interval $(-1,1)$ describe a local presence of a mixture of the two states.

In this work we prove well-posedness results for a problem similar to (1.1), where more general nonlinearities appear. In fact, in our analysis the potential $W$ may be non-smooth and its domain can be different from the whole real line: thus, we admit the so-called singular potentials. Physically meaningful examples of these potentials prescribe $W$ only inside the interval $[-1,1]$, while for the values outside the interval one has $W(u)=+\infty$. Here, we endow our problem with suitable boundary conditions and we deal with two singular potentials, the former applied to $u$ in the interior of $\Omega$, the latter acting on the trace defined on $\Gamma$, linking them by a suitable compatibility condition. Each potential will be the sum of a principal part, assumed to be convex and with possible barriers (like the values \pm 1 ), and of a non-convex perturbation. Moreover, the derivative $W^{\prime}$ in (1.1) has to be understood as a subdifferential $\partial W$ and then the equation has to be read as a differential inclusion, due to the possibly multivalued character of $\partial W$.

A well-known generalization to the Allen-Cahn equation, which models the evolution of $u$ taking into account also the possible variations of the relative temperature of the system (non-isothermal case), has been proposed by Caginalp (see [7]) for melting-solidification processes in several classes of materials. It is obtained by coupling an equation like (1.1) for the order parameter with a heat balance equation for the temperature.

The mathematical literature regarding the Allen-Cahn equation and the Caginalp system is rather vast. In particular, well-posedness results can be found in [20, while the analysis of the dissipative dynamical system generated by these equations has been carried out in a large number of papers. In [16, 17] singular potentials are considered and theorems about the existence of global and/or exponential attractor are proved. We refer the reader also to [8] and [19], where such models are linked to the Cahn-Hilliard equation describing the phase-separation phenomenon, i.e. the progress toward thermodynamic equilibrium of an initial mixture of the phases of the material.

The Allen-Cahn equation and the Caginalp system are usually coupled with homogeneous Neumann boundary conditions for the order parameter. Such conditions are meant to represent the orthogonality of the interface to the boundary and the absence of mass flux. Recently, physicists have introduced the so-called dynamic boundary conditions, using this terminology to underline the fact that the kinetics of the process, i.e. the term $\partial_{t} u$, appears explicitly in the boundary conditions. This particular choice has the advantage of taking into account the interaction of the components of the system with the walls (i.e., within $\Gamma$ ). Dynamic conditions can be associated with equation (1.1) arguing as described in [13. The main idea is to consider a free energy functional in the interior of $\Omega$ and another one defined only on $\Gamma$ in which a sufficiently smooth potential $W_{b}$ appears. One writes that the density on the boundary of the total free energy $E$ relaxes towards 
the equilibrium with a rate proportional to the Fréchet-derivative of $E$ with respect to $u$; then, it is possible to infer that the system is subject to the following dynamic boundary condition

$$
\frac{1}{\delta} \partial_{t} u=\alpha \Delta_{\Gamma} u-\partial_{n} u-\gamma u-W_{b}^{\prime}(u) \text { on } \Gamma \times(0,+\infty)
$$

where the parameters $\delta, \alpha, \gamma$ are positive, $\Delta_{\Gamma}$ is the Laplace-Beltrami operator and $\partial_{n} u$ denotes the outer normal derivative of $u$ on $\Gamma$.

Allen-Cahn equation and Caginalp system endowed with boundary conditions (1.2) have already been examined in some papers by assuming that the potential $W$ has a polynomially controlled growth at most of degree six. Well-posedness results as well as the convergence of the solution to a steady state have been discussed in [10] by means of the Łojasiewicz-Simon technique. In [13]-[14] the authors prove global existence and uniqueness of the solutions and analyze their asymptotic behavior; they show the existence of a global attractor, as well as of an exponential attractor, and convergence to equilibrium as time tends to infinity. In the work of Sprekels and Wu [22] some analogous results are proved together with a suitable Łojasiewicz-Simon type inequality, under the assumptions that the nonlinear terms are real analytic and satisfy some particular growth conditions.

In this paper we are interested to the study of the following problem, where the potentials have been generalized:

$$
\begin{aligned}
& \partial_{t} u-\Delta u+\beta(u)+\pi(u) \ni f \quad \text { in } \Omega \times(0, T), \\
& v:=\left.u\right|_{\Gamma}, \quad \partial_{n} u+\partial_{t} v-\nu \Delta_{\Gamma} v+\beta_{\Gamma}(v)+\pi_{\Gamma}(v) \ni f_{\Gamma} \quad \text { in } \Gamma \times(0, T), \\
& u(0)=u_{0} \text { in } \Omega \quad \text { and } v(0)=v_{0} \text { on } \Gamma .
\end{aligned}
$$

Here, $T$ is an arbitrary positive and finite final time, $\beta$ and $\beta_{\Gamma}$ are maximal monotone and possibly non-smooth graphs, while $\pi$ and $\pi_{\Gamma}$ are non-monotone smooth perturbations. Moreover, we have introduced the nonnegative parameter $\nu$ in (1.4) and added the forcing terms $f$ and $f_{\Gamma}$. We consider nonlinear terms with the following regularities: $\pi$ and $\pi_{\Gamma}$ are Lipschitz continuous functions, while the graphs $\beta$ and $\beta_{\Gamma}$ are naturally subdifferentials of convex functions. Therefore, the equations (1.3) and (1.4) have to be read as differential inclusions. Recalling equation (1.1) endowed with the boundary conditions (1.2), we observe that our choice corresponds to split $W^{\prime}$ and $W_{b}^{\prime}$ as $W^{\prime}=\partial W=\beta+\pi$ and $W_{b}^{\prime}=$ $\partial W_{b}=\beta_{\Gamma}+\pi_{\Gamma}$. A relevant example for $\beta$ and $\beta_{\Gamma}$ is provided by $\beta(u)=\partial I_{[-1,1]}(u)$ and $\beta_{\Gamma}(v)=\partial I_{[-1,1]}(v)$, where the function $I_{[-1,1]}: \mathbb{R} \rightarrow(-\infty,+\infty]$ is the indicator function of the interval $[-1,1]$. We want to obtain well-posedness results for the problem (1.3)(1.5) on any finite time interval $(0, T)$ : our arguments are inspired by those employed by Gilardi, Miranville and Schimperna in [15] when dealing with the Cahn-Hilliard equation.

\subsection{Outline of the paper}

We start our investigation by introducing an equivalent abstract formulation of (1.3) which takes into account both the boundary condition (1.4) and the initial conditions (1.5). After defining the framework in which we will develop our work, we state the continuous dependence on data result (Theorem 2.3) and, as a corollary, the uniqueness result (Theorem 2.4), both holding under quite general assumptions on the data. Existence of 
a weak solution is proved under more restrictive, though natural, assumptions (Theorem 2.5). In particular, we assume that the main nonlinearities $\beta$ and $\beta_{\Gamma}$ satisfy a proper compatibility condition. The existence proof is given using a two-level approximating scheme. Our argument is detailed in Section 4 and runs as follows. First, as the nonlinearities $\beta$ and $\beta_{\Gamma}$ of our problem could be multivalued, we regularize them in order to deal with single-valued and more regular functions. This is what we do at the first level of our approximation by using Yosida approximations depending on a small parameter $\varepsilon$. Then, with the help of a suitable finite-dimensional approximating scheme and thanks to some technical results about the regularizations, we are able to find some a priori estimates on our $\varepsilon$-approximation. By such estimates we can pass to the limit as $\varepsilon \searrow 0$ taking the maximal monotonicity of the graphs into account and by virtue of compactness and monotonicity results. Lastly, Section 5 is dedicated to the analysis of the problem in the special case where the parameter $\nu$ is equal to 0 : here, besides the well-posedness issues, we can prove a convergence result for the solutions of the problem with $\nu>0$ as $\nu \searrow 0$.

\subsection{Notation}

We conclude this section stating a general rule regarding the notation in the paper. In order to avoid a boring notation, throughout the paper the symbols $c$ and $C$ stand for different constants which depend generally on the domain and on the norms of the functions involved. In particular, such constants are independent of the coefficient $\nu$ and the approximation parameter $\varepsilon$ we introduce in next section. On the other hand, a notation like $c_{\delta}$ allows the constant to depend in addition on some parameter $\delta$. Hence, the meaning of $c, C$ and $c_{\delta}$ might change from line to line and even in the same chain of inequalities.

\section{Main results}

Let $\Omega$ be a bounded domain in $\mathbb{R}^{d}, d>1$, with smooth boundary $\Gamma:=\partial \Omega$. We denote by $\partial_{n}$ the outward normal derivative on $\Gamma$. Let $|\Omega|$ stand for the Lebesgue measure of $\Omega$ and let $|\Gamma|$ be the $(d-1)$-dimensional measure of $\Gamma$. Given a final time $T$, we set for convenience

$$
\begin{aligned}
Q_{t}:=\Omega \times(0, t) & \text { and } \quad \Sigma_{t}:=\Gamma \times(0, t) \text { for every } t \in(0, T], \\
Q:=Q_{T} & \text { and } \quad \Sigma:=\Sigma_{T} .
\end{aligned}
$$

We introduce the functions $\hat{\beta}, \hat{\beta}_{\Gamma}, \pi, \pi_{\Gamma}$ and the positive parameter $\nu$ (the case $\nu=0$ will be treated separately) satisfying the following conditions:

$\hat{\beta}, \hat{\beta}_{\Gamma}: \mathbb{R} \rightarrow[0,+\infty)$ are convex, proper, l.s.c., and $\hat{\beta}(0)=\hat{\beta}_{\Gamma}(0)=0$,

$\pi, \pi_{\Gamma}$ are Lipschitz continuous with Lipschitz constants $L, L_{\Gamma}$ respectively, $\nu>0$.

We can therefore consider the subdifferentials $\beta$ and $\beta_{\Gamma}$ in $\mathbb{R} \times \mathbb{R}$ of the functions $\hat{\beta}, \hat{\beta}_{\Gamma}$ :

$$
\beta:=\partial \hat{\beta} \quad \text { and } \quad \beta_{\Gamma}:=\partial \hat{\beta_{\Gamma}},
$$


which turn out to be maximal monotone graphs in $\mathbb{R} \times \mathbb{R}$ (we refer to [2] and [4] for basic definitions and properties of maximal monotone operators). We note that (2.1) entails $\beta(0) \ni 0$ and $\beta_{\Gamma}(0) \ni 0$. In order to let these graphs be as general as possible, we allow them to have effective domains, respectively denoted by

$$
D(\beta)=\{r \in \mathbb{R}: \beta(r) \neq \emptyset\} \quad \text { and } \quad D\left(\beta_{\Gamma}\right)=\left\{r \in \mathbb{R}: \beta_{\Gamma}(r) \neq \emptyset\right\},
$$

possibly different from the whole real line. For $\varepsilon>0$ we introduce also the following notation for any maximal monotone graph $\alpha: \mathbb{R} \rightarrow 2^{\mathbb{R}}$ (see, e.g., [4, p. 28])

$$
\begin{aligned}
& \alpha^{\circ}(r) \text { is the element of } \alpha(r) \text { having minimum modulus, } \\
& \alpha_{\varepsilon}^{Y}:=\varepsilon^{-1}\left(I-(I+\varepsilon \alpha)^{-1}\right) \text { is the Yosida approximation of } \alpha \text { of parameter } \varepsilon \text {. }
\end{aligned}
$$

Just for convenience, we still use the symbol $\alpha$ (and, e.g., $\alpha_{\varepsilon}^{Y}$ as a particular case) to represent the induced maximal monotone operator in $L^{2}(\Omega)$ (more precisely, from $L^{2}(\Omega)$ to $L^{2}(\Omega)$ ), doing the same also for the operators induced in $L^{2}(\Gamma), L^{2}(Q)$ and $L^{2}(\Sigma)$.

As the graphs $\beta$ and $\beta_{\Gamma}$ might be multivalued, in our formulation we introduce two selections $\xi$ and $\xi_{\Gamma}$ from $\beta$ and $\beta_{\Gamma}$, respectively. Therefore, the problem (1.3)-(1.5) can be stated as follows:

$$
\begin{aligned}
& \partial_{t} u-\Delta u+\xi+\pi(u)=f \quad \text { a.e. in } Q \\
& v:=\left.u\right|_{\Gamma}, \quad \partial_{n} u+\partial_{t} v-\nu \Delta_{\Gamma} v+\xi_{\Gamma}+\pi_{\Gamma}(v)=f_{\Gamma} \quad \text { a.e. on } \Sigma, \\
& \xi \in \beta(u) \text { a.e. in } Q \text { and } \xi_{\Gamma} \in \beta_{\Gamma}(v) \text { a.e. on } \Sigma \\
& u(0)=u_{0} \text { a.e. in } \Omega \text { and } v(0)=v_{0} \text { a.e. on } \Gamma \text {. }
\end{aligned}
$$

We point out the functional spaces we will use in the following and set

$$
\begin{aligned}
& V:=H^{1}(\Omega), \quad H:=L^{2}(\Omega), \\
& V_{\Gamma}:=H^{1}(\Gamma), \quad H_{\Gamma}:=L^{2}(\Gamma), \quad \mathcal{V}:=\left\{z \in V:\left.z\right|_{\Gamma} \in V_{\Gamma}\right\} .
\end{aligned}
$$

Note that the inclusion $V \subset H$ is compact and dense. Then we identify $H$ with its dual space $H^{*}$, so that $V \subset H \subset V^{*}$ with dense and compact injections, and therefore $\left(V, H, V^{*}\right)$ is the standard Hilbert triplet. The same considerations hold for $V_{\Gamma}$ and $H_{\Gamma}$. Let $(\cdot, \cdot)$ and $\|\cdot\|_{H}$ be the inner product and the induced norm in $H$. We also denote by $\|\cdot\|_{V},\|\cdot\|_{H_{\Gamma}},\|\cdot\|_{V_{\Gamma}}$ the norms in the corresponding spaces. The symbol $\langle\cdot, \cdot\rangle$ stands for the duality pairing between $V^{*}$ and $V$. We endow the space $\mathcal{V} \subset V$ with the following inner product and the induced graph norm:

$$
(w, z)_{\mathcal{V}}:=(w, z)_{V}+\left(\left.w\right|_{\Gamma},\left.z\right|_{\Gamma}\right)_{V_{\Gamma}}, \quad\|w\|_{\mathcal{V}}^{2}=\|w\|_{V}^{2}+\left\|\left.w\right|_{\Gamma}\right\|_{V_{\Gamma}}^{2}, \quad w, z \in \mathcal{V} .
$$

We present now the variational formulation of the problem (2.6)-(2.9) in the abstract setting introduced above. In order to obtain it, we formally multiply the equation (2.6) by test functions and integrate by parts on $\Omega$ taking the boundary conditions (2.7) into account. We have to make precise the regularity of all the components of the problem: the results of continuous dependence and uniqueness of the solution hold under quite general assumptions, while further requirements have to be specified in order to prove the existence result. So, just to start, we ask the data to fulfill the conditions

$$
f \in L^{2}(0, T ; H), \quad f_{\Gamma} \in L^{2}\left(0, T ; H_{\Gamma}\right), \quad u_{0} \in H \quad \text { and } \quad v_{0} \in H_{\Gamma}
$$


and look for a quadruplet $\left(u, v, \xi, \xi_{\Gamma}\right)$ such that

$$
\begin{aligned}
& u \in L^{2}(0, T ; V) \cap H^{1}\left(0, T ; V^{*}\right), \\
& v:=\left.u\right|_{\Gamma} \in L^{2}\left(0, T ; V_{\Gamma}\right) \cap H^{1}\left(0, T ; V_{\Gamma}^{*}\right), \\
& \xi \in L^{2}(0, T ; H) \equiv L^{2}(Q) \text { and } \xi \in \beta(u) \text { a.e. in } Q, \\
& \xi_{\Gamma} \in L^{2}\left(0, T ; H_{\Gamma}\right) \equiv L^{2}(\Sigma) \text { and } \xi_{\Gamma} \in \beta_{\Gamma}(v) \text { a.e. on } \Sigma, \\
& u(0)=u_{0}, v(0)=v_{0}
\end{aligned}
$$

and satisfying for a.a. $t \in(0, T)$ and every $z \in \mathcal{V}$

$$
\begin{aligned}
& \left\langle\partial_{t} u(t), z\right\rangle+\int_{\Omega} \nabla u(t) \cdot \nabla z+\int_{\Omega}(\xi(t)+\pi(u(t))) z+V_{\Gamma}^{*}\left\langle\partial_{t} v(t), z\right\rangle_{V_{\Gamma}} \\
& +\nu \int_{\Gamma} \nabla_{\Gamma} v(t) \cdot \nabla_{\Gamma} z+\int_{\Gamma}\left(\xi_{\Gamma}(t)+\pi_{\Gamma}(v(t))\right) z=\int_{\Omega} f(t) z+\int_{\Gamma} f_{\Gamma}(t) z .
\end{aligned}
$$

Remark 2.1. With a little abuse of notation we will use the same letter $z$ to denote the function $z \in \mathcal{V}$ and its trace $\left.z\right|_{\Gamma} \in V_{\Gamma}$. If $z \in L^{2}(0, T ; V)$, the meaning of $\partial_{t} z$ on the boundary has to be understood as $\partial_{t}\left(\left.z\right|_{\Gamma}\right)$ which exists at least in a distributional sense.

Remark 2.2. We observe that regularities (2.13) and (2.14) imply that

$$
u \in C^{0}([0, T] ; H) \quad \text { and } \quad v \in C^{0}\left([0, T] ; H_{\Gamma}\right) .
$$

Thus, in particular, the values $u(t)$ and $v(t)$ make sense in $H$ and $H_{\Gamma}$, respectively, for all $t \in[0, T]$, and this gives a meaning to the initial conditions (2.17) in such spaces.

In the framework specified by (2.12)-(2.16), the following continuous dependence result holds true, together with a uniqueness one as its immediate consequence.

Theorem 2.3 (Continuous dependence on the data). Assume (2.1)-(2.4), (2.12) and let $\left(u_{1}, v_{1}\right),\left(u_{2}, v_{2}\right)$ be the first two components of two different solutions of the problem (2.13) -(2.18), each one corresponding to a set of data $u_{0, i}, v_{0, i}, f_{i}, f_{\Gamma, i}$ with $i=1,2$. Then, there is a constant $c$ such that

$$
\begin{aligned}
& \left\|u_{1}-u_{2}\right\|_{L^{\infty}(0, T ; H)}^{2}+\left\|\nabla\left(u_{1}-u_{2}\right)\right\|_{L^{2}(Q)}^{2} \\
& +\left\|v_{1}-v_{2}\right\|_{L^{\infty}\left(0, T ; H_{\Gamma}\right)}^{2}+\nu\left\|\nabla_{\Gamma}\left(v_{1}-v_{2}\right)\right\|_{L^{2}(\Sigma)}^{2} \\
& \quad \leq c\left\{\left\|u_{0,1}-u_{0,2}\right\|_{H}^{2}+\left\|v_{0,1}-v_{0,2}\right\|_{H_{\Gamma}}^{2}\right. \\
& \left.\quad+\left\|f_{1}-f_{2}\right\|_{L^{2}(0, T ; H)}^{2}+\left\|f_{\Gamma, 1}-f_{\Gamma, 2}\right\|_{L^{2}\left(0, T ; H_{\Gamma}\right)}^{2}\right\} .
\end{aligned}
$$

Theorem 2.4 (Uniqueness). Under the same assumptions as in Theorem 2.3, then any two solutions of the problem (2.13) $-(2.18)$ coincide.

In order to prove the existence of the solution, we have to enforce our assumptions requiring:

$$
\begin{aligned}
& u_{0} \in V, \quad v_{0}=\left.u_{0}\right|_{\Gamma} \in V_{\Gamma} \\
& \hat{\beta}\left(u_{0}\right) \in L^{1}(\Omega) \text { and } \hat{\beta}_{\Gamma}\left(v_{0}\right) \in L^{1}(\Gamma) .
\end{aligned}
$$


Of course, (2.20) is equivalent to $u_{0} \in \mathcal{V}$ and the trace of $u_{0}$ on the boundary coincides with the initial value $v_{0}$. Moreover, we introduce also a compatibility condition on the main nonlinearities $\beta$ and $\beta_{\Gamma}$. Namely, we assume that:

$$
D(\beta) \supseteq D\left(\beta_{\Gamma}\right)
$$

and that two real constants $\eta$ and $C_{\Gamma}$ exist such that

$$
\eta>0, \quad C_{\Gamma} \geq 0, \quad\left|\beta^{\circ}(r)\right| \leq \eta\left|\beta_{\Gamma}^{\circ}(r)\right|+C_{\Gamma} \text { for every } r \in D\left(\beta_{\Gamma}\right) .
$$

We state now our main result, whose proof is given in Section 4.

Theorem 2.5 (Existence). Assume (2.1) -(2.4), (2.12) and (2.20)-(2.23). Then, there exists a quadruplet $\left(u, v, \xi, \xi_{\Gamma}\right)$ solving problem (2.13)-(2.18). Moreover, $u$ and $v$ fulfill

$$
u \in L^{\infty}(0, T ; V) \cap H^{1}(0, T ; H), \quad v \in L^{\infty}\left(0, T ; V_{\Gamma}\right) \cap H^{1}\left(0, T ; H_{\Gamma}\right) .
$$

In Section 5 we prove that very similar continuous dependence and existence results hold in the case $\nu=0$ (see Theorems 5.1 and 5.3). As we will point out, in order to do this we have to change our choice of the functional spaces on the boundary and extend in a suitable way the operator related to $\beta_{\Gamma}$. Moreover, we will be able to show that the solution of the problem for $\nu>0$ converges in a suitable topology to the solution of the problem with $\nu=0$.

\section{Continuous dependence on the data}

This section contains the proofs of Theorems 2.3 and 2.4. In order to prove Theorem 2.3 we consider two different solutions of the problem (2.13)-(2.18) coming each from a different set of data and we label their components with subscripts 1 and 2. We write (2.18) for both solutions and take the difference, defining $u$ as $u:=u_{1}-u_{2}$ and doing the same for $v:=v_{1}-v_{2}, \xi:=\xi_{1}-\xi_{2}, \xi_{\Gamma}:=\xi_{\Gamma, 1}-\xi_{\Gamma, 2}$. Then, we write such a difference at time $t=s$, we choose as test-function $z \in \mathcal{V}$ the function $u(s)=u_{1}(s)-u_{2}(s)$ itself and integrate what we get over $(0, t)$, where $t \in(0, T]$ is arbitrary. We obtain

$$
\begin{aligned}
& \frac{1}{2}\|u(t)\|_{H}^{2}+\frac{1}{2}\|v(t)\|_{H_{\Gamma}}^{2}-\frac{1}{2}\left\|u_{0,1}-u_{0,2}\right\|_{H}^{2}-\frac{1}{2}\left\|v_{0,1}-v_{0,2}\right\|_{H_{\Gamma}}^{2} \\
& \quad+\int_{Q_{t}}|\nabla u|^{2}+\nu \int_{\Sigma_{t}}\left|\nabla_{\Gamma} v\right|^{2}+\int_{Q_{t}} \xi u+\int_{\Sigma_{t}} \xi_{\Gamma} v \\
& =\int_{Q_{t}}\left(\pi\left(u_{2}\right)-\pi\left(u_{1}\right)\right) u+\int_{\Sigma_{t}}\left(\pi_{\Gamma}\left(v_{2}\right)-\pi_{\Gamma}\left(v_{1}\right)\right) v \\
& \quad+\int_{Q_{t}}\left(f_{1}-f_{2}\right) u+\int_{\Sigma_{t}}\left(f_{\Gamma, 1}-f_{\Gamma, 2}\right) v .
\end{aligned}
$$

The last two terms on the left-hand side of (3.1) are nonnegative, due to the monotonicity properties of $\beta$ and $\beta_{\Gamma}$. As far as the right-hand side is concerned, we can estimate the first two terms with the help of $(2.2)$ :

$$
\int_{Q_{t}}\left(\pi\left(u_{2}\right)-\pi\left(u_{1}\right)\right) u+\int_{\Sigma_{t}}\left(\pi_{\Gamma}\left(v_{2}\right)-\pi_{\Gamma}\left(v_{1}\right)\right) v \leq L \int_{Q_{t}}|u|^{2}+L_{\Gamma} \int_{\Sigma_{t}}|v|^{2} .
$$


For the other two terms, we simply use Young's inequality:

$$
\begin{aligned}
& \int_{Q_{t}}\left(f_{1}-f_{2}\right) u+\int_{\Sigma_{t}}\left(f_{\Gamma, 1}-f_{\Gamma, 2}\right) v \\
& \leq \frac{1}{2} \int_{Q_{t}}\left|f_{1}-f_{2}\right|^{2}+\frac{1}{2} \int_{Q_{t}}|u|^{2}+\frac{1}{2} \int_{\Sigma_{t}}\left|f_{\Gamma, 1}-f_{\Gamma, 2}\right|^{2}+\frac{1}{2} \int_{\Sigma_{t}}|v|^{2} .
\end{aligned}
$$

Thus, thanks to (3.1)- (3.3) we have

$$
\begin{aligned}
& \|u(t)\|_{H}^{2}+\|v(t)\|_{H_{\Gamma}}^{2}+\|\nabla u\|_{L^{2}\left(Q_{t}\right)}^{2}+\nu\left\|\nabla_{\Gamma} v\right\|_{L^{2}\left(\Sigma_{t}\right)}^{2} \\
& \leq \int_{Q_{t}}\left|f_{1}-f_{2}\right|^{2}+\int_{\Sigma_{t}}\left|f_{\Gamma, 1}-f_{\Gamma, 2}\right|^{2}+(2 L+1) \int_{Q_{t}}|u|^{2}+\left(2 L_{\Gamma}+1\right) \int_{\Sigma_{t}}|v|^{2} \\
& +\left\|u_{0,1}-u_{0,2}\right\|_{H}^{2}+\left\|v_{0,1}-v_{0,2}\right\|_{H_{\Gamma}}^{2} .
\end{aligned}
$$

We can now apply the Gronwall Lemma and then take the supremum over the interval $(0, T)$ thus finding $L^{\infty}$-in time estimates for the left-hand side of this inequality. Scaling the constants, we finally get the estimate (2.19).

For the proof of Theorem 2.4, we note that Theorem 2.3 yields the uniqueness of the first two components of the solution, that is, $u_{1}=u_{2}$ and $v_{1}=v_{2}$. Then, with the help of (2.18) we easily obtain

$$
\int_{\Omega}\left(\xi_{1}-\xi_{2}\right)(t) z+\int_{\Gamma}\left(\xi_{\Gamma, 1}-\xi_{\Gamma, 2}\right)(t) z=0 \quad \text { for all } z \in \mathcal{V}, \quad \text { for a.a. } t \in(0, T) .
$$

Taking first $z \in H_{0}^{1}(\Omega)(\subset \mathcal{V})$, we easily deduce that $\xi_{1}=\xi_{2}$; then, by rewriting (3.4) for a general test function $z \in \mathcal{V}$, we can conclude that $\xi_{\Gamma, 1}=\xi_{\Gamma, 2}$.

\section{Existence}

This section is devoted to the proof of Theorem [2.5. We fix a parameter $\varepsilon \in(0,1)$ and consider an approximating problem depending on $\varepsilon$, obtained by regularizing the nonlinearities $\beta$ and $\beta_{\Gamma}$. To this aim, we define the functions $\beta_{\varepsilon}$ and $\beta_{\Gamma, \varepsilon}: \mathbb{R} \rightarrow \mathbb{R}$ by

$$
\begin{aligned}
& \beta_{\varepsilon}(r):=\beta_{\varepsilon}^{Y}(r) \text { for } r \in \mathbb{R}, \\
& \beta_{\Gamma, \varepsilon}(r):=\left(\beta_{\Gamma}\right)_{\varepsilon \eta}^{Y}(r) \quad \text { for } r \in \mathbb{R} .
\end{aligned}
$$

Here, we have used the notation (2.5) for the Yosida approximations and the parameter $\eta$ is the fixed constant given by (2.23). The choice of a function $\beta_{\Gamma, \varepsilon}$ as above is justified by the forthcoming Lemma 4.4 which ensures a compatibility condition similar to (2.23).

For convenience, we define also the functions $\hat{\beta}_{\varepsilon}, \hat{\beta}_{\Gamma, \varepsilon}: \mathbb{R} \rightarrow \mathbb{R}$ by

$$
\hat{\beta}_{\varepsilon}(r):=\int_{0}^{r} \beta_{\varepsilon}(s) d s \quad \text { and } \quad \hat{\beta}_{\Gamma, \varepsilon}(r):=\int_{0}^{r} \beta_{\Gamma, \varepsilon}(s) d s \text { for } r \in \mathbb{R} .
$$

Let us recall that the Yosida $\lambda$-regularization of a maximal monotone operator is monotone and Lipschitz continuous with $1 / \lambda$ as Lipschitz constant (see 4, Propositions 2.6 
and 2.7]). Thus, such a property holds in particular for $\beta_{\Gamma, \varepsilon}$ with $1 / \varepsilon \eta$ as Lipschitz constant. Moreover, both $\beta_{\varepsilon}$ and $\beta_{\Gamma, \varepsilon}$ vanish at 0 . It follows that $\hat{\beta}_{\varepsilon}$ and $\hat{\beta}_{\Gamma, \varepsilon}$ are nonnegative convex functions with (at most) quadratic growth. We finally point out a general property of Yosida approximations of every maximal monotone operator (see [4, Prop. 2.6, p. 28]):

$$
\begin{array}{r}
\left|\beta_{\varepsilon}(r)\right| \leq\left|\beta^{\circ}(r)\right| \text { for } r \in D(\beta), \quad\left|\beta_{\Gamma, \varepsilon}(r)\right| \leq\left|\beta_{\Gamma}^{\circ}(r)\right| \text { for } r \in D\left(\beta_{\Gamma}\right), \\
0 \leq \hat{\beta}_{\varepsilon}(r) \leq \hat{\beta}(r) \text { and } 0 \leq \hat{\beta}_{\Gamma, \varepsilon}(r) \leq \hat{\beta}_{\Gamma}(r) \text { for all } r \in \mathbb{R} .
\end{array}
$$

Our approximating problem consists in finding a function $u_{\varepsilon}$ satisfying

$$
\begin{aligned}
& u_{\varepsilon} \in L^{\infty}(0, T ; V) \cap H^{1}(0, T ; H) \\
& \left.u_{\varepsilon}\right|_{\Gamma} \in L^{\infty}\left(0, T ; V_{\Gamma}\right) \cap H^{1}\left(0, T ; H_{\Gamma}\right) \\
& u_{\varepsilon}(0)=u_{0}
\end{aligned}
$$

and solving for a.a. $t \in(0, T)$ the variational equality

$$
\begin{aligned}
& \int_{\Omega} \partial_{t} u_{\varepsilon}(t) z+\int_{\Omega} \nabla u_{\varepsilon}(t) \cdot \nabla z+\int_{\Omega}\left(\beta_{\varepsilon}+\pi\right)\left(u_{\varepsilon}(t)\right) z+\int_{\Gamma} \partial_{t} u_{\varepsilon}(t) z \\
& +\nu \int_{\Gamma} \nabla_{\Gamma} u_{\varepsilon}(t) \cdot \nabla_{\Gamma} z+\int_{\Gamma}\left(\beta_{\Gamma, \varepsilon}+\pi_{\Gamma}\right)\left(u_{\varepsilon}(t)\right) z=\int_{\Omega} f(t) z+\int_{\Gamma} f_{\Gamma}(t) z
\end{aligned}
$$

for every $z \in \mathcal{V}$, where, referring to Remark 2.1, from now on we simply write $u_{\varepsilon}$ instead of $\left.u_{\varepsilon}\right|_{\Gamma}$ to indicate the trace of $u_{\varepsilon}$ on the boundary.

We state our well-posedness result for the above problem.

Proposition 4.1. Assume (2.2), (2.12) and (2.20). Let $\beta_{\varepsilon}$ and $\beta_{\Gamma, \varepsilon}: \mathbb{R} \rightarrow \mathbb{R}$ be the functions introduced in (4.1)-(4.2). Then, there exists a unique function $u_{\varepsilon}$ satisfying (4.5) $-(4.8)$.

The uniqueness proof follows as a particular case of Theorem 2.4. In order to show the existence of the solution, we need to introduce an approximation of our $\varepsilon$-problem in a finite dimensional space using a standard Faedo-Galerkin technique. Hence, we can show that a more regular discrete solution $u_{\varepsilon}^{n}$ exists by virtue of well-known results for systems of ordinary differential equations. We can thus find the desired $u_{\varepsilon}$ by passing to the limit as $n \nearrow+\infty$ and exploiting some compactness and monotonicity results (see [15] and [9] for further details).

\subsection{Estimates}

We perform now some a priori estimates on $u_{\varepsilon}, \beta_{\varepsilon}\left(u_{\varepsilon}\right), \beta_{\Gamma, \varepsilon}\left(u_{\varepsilon}\right)$ in order to solve the problem (2.13)-(2.18) by letting $\varepsilon$ tend to 0 . All such estimates can be rigorously justified at the level of the Galerkin scheme (see [9]). 


\subsubsection{First a priori estimate}

We choose $z=u_{\varepsilon}(t)$ in (4.8): we are allowed to do this thanks to the regularities (4.5)(4.6). With the help of Young's inequality we obtain

$$
\begin{aligned}
& \frac{1}{2} \frac{d}{d t}\left\|u_{\varepsilon}(t)\right\|_{H}^{2}+\frac{1}{2} \frac{d}{d t}\left\|u_{\varepsilon}(t)\right\|_{H_{\Gamma}}^{2}+\left\|\nabla u_{\varepsilon}(t)\right\|_{H}^{2}+\nu\left\|\nabla_{\Gamma} u_{\varepsilon}(t)\right\|_{H_{\Gamma}}^{2} \\
& +\int_{\Omega} \beta_{\varepsilon}\left(u_{\varepsilon}(t)\right) u_{\varepsilon}(t)+\int_{\Gamma} \beta_{\Gamma, \varepsilon}\left(u_{\varepsilon}(t)\right) u_{\varepsilon}(t) \leq-\int_{\Omega} \pi\left(u_{\varepsilon}(t)\right) u_{\varepsilon}(t)-\int_{\Gamma} \pi_{\Gamma}\left(u_{\varepsilon}(t)\right) u_{\varepsilon}(t) \\
& \quad+\frac{1}{2}\|f(t)\|_{H}^{2}+\frac{1}{2}\left\|f_{\Gamma}(t)\right\|_{H_{\Gamma}}^{2}+\frac{1}{2}\left\|u_{\varepsilon}(t)\right\|_{H}^{2}+\frac{1}{2}\left\|u_{\varepsilon}(t)\right\|_{H_{\Gamma}}^{2}
\end{aligned}
$$

The two terms containing $\beta_{\varepsilon}$ and $\beta_{\Gamma, \varepsilon}$ are nonnegative since both $\beta_{\varepsilon}$ and $\beta_{\Gamma, \varepsilon}$ are monotone and null in 0 , so we can avoid considering them. On the other hand, due to (2.2) we have that

$$
\begin{aligned}
& -\int_{\Omega} \pi\left(u_{\varepsilon}(t)\right) u_{\varepsilon}(t)-\int_{\Gamma} \pi_{\Gamma}\left(u_{\varepsilon}(t)\right) u_{\varepsilon}(t) \\
& \leq(L+1) \int_{\Omega}\left|u_{\varepsilon}(t)\right|^{2}+\left(L_{\Gamma}+1\right) \int_{\Gamma}\left|u_{\varepsilon}(t)\right|^{2}+C,
\end{aligned}
$$

where the constant $C$ depends just on $|\Omega|,|\Gamma|$ and on the values taken in 0 by the functions $\pi$ and $\pi_{\Gamma}$. Then, integrating both sides of (4.9) with respect to time and applying the Gronwall lemma, we easily find that

$$
\begin{aligned}
& \left\|u_{\varepsilon}(t)\right\|_{H}^{2}+\left\|u_{\varepsilon}(t)\right\|_{H_{\Gamma}}^{2}+\left\|\nabla u_{\varepsilon}\right\|_{L^{2}(0, t ; H)}^{2}+\nu\left\|\nabla_{\Gamma} u_{\varepsilon}\right\|_{L^{2}\left(0, t ; H_{\Gamma}\right)}^{2} \\
& \leq C\left(1+\left\|u_{0}\right\|_{H}^{2}+\left\|u_{0}\right\|_{H_{\Gamma}}^{2}+\|f\|_{L^{2}(0, T ; H)}^{2}+\left\|f_{\Gamma}\right\|_{L^{2}\left(0, T ; H_{\Gamma}\right)}^{2}\right)
\end{aligned}
$$

for all $t \in(0, T)$. Therefore, in view of (2.12), it turns out that

$$
\left\|u_{\varepsilon}\right\|_{L^{\infty}(0, T ; H) \cap L^{2}(0, T ; V)}+\left\|u_{\varepsilon}\right\|_{L^{\infty}\left(0, T ; H_{\Gamma}\right)}+\nu^{1 / 2}\left\|u_{\varepsilon}\right\|_{L^{2}\left(0, T ; V_{\Gamma}\right)} \leq C .
$$

\subsubsection{Second a priori estimate}

We have now to proceed formally by testing equation (4.8) by $z=\partial_{t} u_{\varepsilon}(t)$ and integrating with respect to $t$. Owing to (4.3), we obtain

$$
\begin{aligned}
& \int_{Q_{t}}\left|\partial_{t} u_{\varepsilon}\right|^{2}+\int_{\Sigma_{t}}\left|\partial_{t} u_{\varepsilon}\right|^{2}+\frac{1}{2} \int_{\Omega}\left|\nabla u_{\varepsilon}(t)\right|^{2} \\
& +\frac{\nu}{2} \int_{\Gamma}\left|\nabla_{\Gamma} u_{\varepsilon}(t)\right|^{2}+\int_{\Omega} \hat{\beta}_{\varepsilon}\left(u_{\varepsilon}(t)\right)+\int_{\Gamma} \hat{\beta}_{\Gamma, \varepsilon}\left(u_{\varepsilon}(t)\right) \\
& \leq \frac{1}{2} \int_{\Omega}\left|\nabla u_{0}\right|^{2}+\frac{\nu}{2} \int_{\Gamma}\left|\nabla_{\Gamma} u_{0}\right|^{2}+\int_{\Omega} \hat{\beta}_{\varepsilon}\left(u_{0}\right)+\int_{\Gamma} \hat{\beta}_{\Gamma, \varepsilon}\left(u_{0}\right) \\
& \quad+\int_{Q_{t}}\left(f-\pi\left(u_{\varepsilon}\right)\right) \partial_{t} u_{\varepsilon}+\int_{\Sigma_{t}}\left(f_{\Gamma}-\pi_{\Gamma}\left(u_{\varepsilon}\right)\right) \partial_{t} u_{\varepsilon} .
\end{aligned}
$$

As all the integrals on the left-hand side are nonnegative, we consider the ones on the right-hand side. Owing to (2.12), (2.2) and (4.11), by applying Young's inequality we deduce that

$$
\int_{Q_{t}}\left(f-\pi\left(u_{\varepsilon}\right)\right) \partial_{t} u_{\varepsilon}+\int_{\Sigma_{t}}\left(f_{\Gamma}-\pi_{\Gamma}\left(u_{\varepsilon}\right)\right) \partial_{t} u_{\varepsilon} \leq C+\frac{1}{2} \int_{Q_{t}}\left|\partial_{t} u_{\varepsilon}\right|^{2}+\frac{1}{2} \int_{\Sigma_{t}}\left|\partial_{t} u_{\varepsilon}\right|^{2} .
$$


Next, we examine the terms containing the initial conditions $u_{0}$. Thanks to the general property pointed out in (4.4), we have

$$
\int_{\Omega} \hat{\beta}_{\varepsilon}\left(u_{0}\right)+\int_{\Gamma} \hat{\beta}_{\Gamma, \varepsilon}\left(u_{0}\right) \leq \int_{\Omega} \hat{\beta}\left(u_{0}\right)+\int_{\Gamma} \hat{\beta}_{\Gamma}\left(u_{0}\right)
$$

and (2.20)-(2.21) allow us to infer

$$
\frac{1}{2} \int_{\Omega}\left|\nabla u_{0}\right|^{2}+\frac{\nu}{2} \int_{\Gamma}\left|\nabla_{\Gamma} u_{0}\right|^{2}+\int_{\Omega} \hat{\beta}_{\varepsilon}\left(u_{0}\right)+\int_{\Gamma} \hat{\beta}_{\Gamma, \varepsilon}\left(u_{0}\right) \leq C .
$$

Then, from (4.12) we conclude that

$$
\left\|u_{\varepsilon}\right\|_{L^{\infty}(0, T ; V) \cap H^{1}(0, T ; H)}+\left\|u_{\varepsilon}\right\|_{H^{1}\left(0, T ; H_{\Gamma}\right)}+\nu^{1 / 2}\left\|u_{\varepsilon}\right\|_{L^{\infty}\left(0, T ; V_{\Gamma}\right)} \leq C .
$$

\subsubsection{Preliminary results to the following estimates}

We highlight now some general properties of the Yosida approximation that will be useful to prove the subsequent Lemma 4.4.

Lemma 4.2. Let $\gamma: \mathbb{R} \rightarrow 2^{\mathbb{R}}$ be a maximal monotone graph and let $\varepsilon, b>0$. Then

$$
(b \gamma)_{\varepsilon}^{Y}=b(\gamma)_{b \varepsilon}^{Y}
$$

Proof. Recalling the definition of Yosida approximation given by (2.5), the equalities

$$
b(\gamma)_{b \varepsilon}^{Y}=b \frac{1}{b \varepsilon}\left(I-(I+b \varepsilon \gamma)^{-1}\right)=\frac{1}{\varepsilon}\left(I-(I+\varepsilon(b \gamma))^{-1}\right)=(b \gamma)_{\varepsilon}^{Y} .
$$

enable us to deduce (4.14).

Lemma 4.3. Let $\gamma: \mathbb{R} \rightarrow 2^{\mathbb{R}}$ be a maximal monotone graph, $a \in \mathbb{R}, \varepsilon>0$. Then

$$
(\gamma+a)_{\varepsilon}^{Y}(r)=\gamma_{\varepsilon}^{Y}(r-\varepsilon a)+a \quad \text { for every } r \in \mathbb{R} .
$$

Proof. Recalling the definitions of Yosida approximation and of the inverse operator of a multivalued operator (see, e.g., [2, 4]), for $r \in \mathbb{R}$ we have that

$$
(\gamma+a)_{\varepsilon}^{Y}(r)=\frac{1}{\varepsilon}\left(I-(I+\varepsilon(\gamma+a))^{-1}\right)(r)=\frac{r}{\varepsilon}-\frac{y}{\varepsilon},
$$

where $y$ is such that $r \in y+\varepsilon(\gamma+a)(y)=y+\varepsilon \gamma(y)+\varepsilon a$. We infer

$$
r-\varepsilon a \in y+\varepsilon \gamma(y),
$$

which is equivalent to $y=(I+\varepsilon \gamma)^{-1}(r-\varepsilon a)$. On the other hand, we have

$$
\gamma_{\varepsilon}^{Y}(r-\varepsilon a)+a=\frac{1}{\varepsilon}\left(I-(I+\varepsilon \gamma)^{-1}\right)(r-\varepsilon a)+a=\frac{r}{\varepsilon}-a+\frac{1}{\varepsilon} y+a=\frac{r}{\varepsilon}-\frac{y}{\varepsilon},
$$

whence the thesis follows from (4.15). 
Now, in view of definitions (4.1) and (4.2), we prove that a variation of (2.23) holds true at the $\varepsilon$-level for our Yosida regularizations. Then, we will take advantage of it in the following estimates.

Lemma 4.4. There holds

$$
\left|\beta_{\varepsilon}(r)\right| \leq \eta\left|\beta_{\Gamma, \varepsilon}(r)\right|+C_{\Gamma} \quad \text { for every } r \in \mathbb{R}
$$

Proof. It is trivial to check (4.16) for $r=0$. Then, we distinguish the cases $r>0$ and $r<0$. We observe that (2.1) and (2.22) imply that $r, \beta_{\Gamma}^{\circ}(r)$ and $\beta^{\circ}(r)$ have the same sign for every $r \in D\left(\beta_{\Gamma}\right)$. If $r>0$ and $r \in D\left(\beta_{\Gamma}\right)$, we can multiply inequality (2.23) by $\varepsilon(>0)$ and then add to both sides $r$, thus obtaining the equivalent inequality

$$
r+\varepsilon \beta^{\circ}(r) \leq \varepsilon\left(\eta \beta_{\Gamma}^{\circ}(r)+C_{\Gamma}\right)+r
$$

which, in terms of operators, can be rewritten as $\left(I+\varepsilon \beta^{\circ}\right)(r) \leq\left(I+\varepsilon\left(\eta \beta_{\Gamma}^{\circ}+C_{\Gamma}\right)\right)(r)$. Hence, thanks to the maximal monotonicity of the graphs $\beta$ and $\eta \beta_{\Gamma}+C_{\Gamma}$, we infer that for their resolvent operators the following inequality holds:

$$
(I+\varepsilon \beta)^{-1}(s) \geq\left(I+\varepsilon\left(\eta \beta_{\Gamma}+C_{\Gamma}\right)\right)^{-1}(s) \text { for every } s>\varepsilon C_{\Gamma} .
$$

Recalling the definition of Yosida regularization of a maximal monotone graph given in (2.5), we observe that (4.17) reduces to

$$
\left(I-\varepsilon \beta_{\varepsilon}^{Y}\right)(s) \geq\left(I-\varepsilon\left(\eta \beta_{\Gamma}+C_{\Gamma}\right)_{\varepsilon}^{Y}\right)(s),
$$

whence we obtain

$$
\beta_{\varepsilon}^{Y}(s) \leq\left(\eta \beta_{\Gamma}+C_{\Gamma}\right)_{\varepsilon}^{Y}(s) .
$$

Applying Lemma 4.3 and Lemma 4.2 to the right-hand side of the above inequality, we deduce that

$$
\left(\eta \beta_{\Gamma}+C_{\Gamma}\right)_{\varepsilon}^{Y}(s)=\left(\eta \beta_{\Gamma}\right)_{\varepsilon}^{Y}\left(s-\varepsilon C_{\Gamma}\right)+C_{\Gamma}=\eta\left(\beta_{\Gamma}\right)_{\varepsilon \eta}^{Y}\left(s-\varepsilon C_{\Gamma}\right)+C_{\Gamma}
$$

and consequently

$$
\beta_{\varepsilon}^{Y}(s) \leq \eta\left(\beta_{\Gamma}\right)_{\varepsilon \eta}^{Y}\left(s-\varepsilon C_{\Gamma}\right)+C_{\Gamma} \quad \text { for every } s>\varepsilon C_{\Gamma} .
$$

Hence, we infer

$$
\beta_{\varepsilon}^{Y}\left(s+\varepsilon C_{\Gamma}\right) \leq \eta\left(\beta_{\Gamma}\right)_{\varepsilon \eta}^{Y}(s)+C_{\Gamma} \quad \text { for every } s>0 .
$$

At this point, we consider the left-hand side of (4.19) and note that, by the monotonicity of $\beta_{\varepsilon}^{Y}, \beta_{\varepsilon}^{Y}(s) \leq \beta_{\varepsilon}^{Y}\left(s+\varepsilon C_{\Gamma}\right)$. Then, owing to (4.1)-(4.2), we have that

$$
\beta_{\varepsilon}(s) \leq \eta \beta_{\Gamma, \varepsilon}(s)+C_{\Gamma}
$$

for every $s>0$. Arguing similarly for $r<0$, we finally show (4.16). 


\subsubsection{Third a priori estimate}

We test now equation (4.8) by $\beta_{\varepsilon}\left(u_{\varepsilon}(t)\right)$ with $t \in(0, T)$, then we integrate over $(0, T)$. Letting $\delta$ be an arbitrary positive number and adding the positive term $\delta \int_{\Sigma}\left|\beta_{\varepsilon}\left(u_{\varepsilon}\right)\right|^{2}$ to both sides for convenience, we rearrange and obtain

$$
\begin{aligned}
& \int_{\Omega} \hat{\beta}_{\varepsilon}\left(u_{\varepsilon}(T)\right)+\int_{\Gamma} \hat{\beta}_{\varepsilon}\left(u_{\varepsilon}(T)\right)+\int_{Q} \beta_{\varepsilon}^{\prime}\left(u_{\varepsilon}\right)\left|\nabla u_{\varepsilon}\right|^{2} \\
& +\nu \int_{\Sigma} \beta_{\varepsilon}^{\prime}\left(u_{\varepsilon}\right)\left|\nabla_{\Gamma} u_{\varepsilon}\right|^{2}+\int_{Q}\left|\beta_{\varepsilon}\left(u_{\varepsilon}\right)\right|^{2}+\delta \int_{\Sigma}\left|\beta_{\varepsilon}\left(u_{\varepsilon}\right)\right|^{2} \\
& =\int_{\Omega} \hat{\beta}_{\varepsilon}\left(u_{0}\right)+\int_{\Gamma} \hat{\beta}_{\varepsilon}\left(u_{0}\right)+\int_{Q}\left(f-\pi\left(u_{\varepsilon}\right)\right) \beta_{\varepsilon}\left(u_{\varepsilon}\right) \\
& +\int_{\Sigma}\left(f_{\Gamma}-\pi_{\Gamma}\left(u_{\varepsilon}\right)\right) \beta_{\varepsilon}\left(u_{\varepsilon}\right)+\int_{\Sigma}\left(\delta\left|\beta_{\varepsilon}\left(u_{\varepsilon}\right)\right|^{2}-\beta_{\varepsilon}\left(u_{\varepsilon}\right) \beta_{\Gamma, \varepsilon}\left(u_{\varepsilon}\right)\right) .
\end{aligned}
$$

The first two terms on the right-hand side of (4.20) can be easily treated owing to (4.4) and (2.21), thus obtaining

$$
\int_{\Omega} \hat{\beta}_{\varepsilon}\left(u_{0}\right)+\int_{\Gamma} \hat{\beta}_{\varepsilon}\left(u_{0}\right) \leq C .
$$

In view of (2.12), (2.2) and (4.11), by Young's inequality we infer that

$$
\int_{Q}\left(f-\pi\left(u_{\varepsilon}\right)\right) \beta_{\varepsilon}\left(u_{\varepsilon}\right) \leq C+\frac{1}{2}\left\|\beta_{\varepsilon}\left(u_{\varepsilon}\right)\right\|_{L^{2}(0, T ; H)}^{2}
$$

and

$$
\int_{\Sigma}\left(f_{\Gamma}-\pi_{\Gamma}\left(u_{\varepsilon}\right)\right) \beta_{\varepsilon}\left(u_{\varepsilon}\right) \leq \frac{\delta}{2}\left\|\beta_{\varepsilon}\left(u_{\varepsilon}\right)\right\|_{L^{2}\left(0, T ; H_{\Gamma}\right)}^{2}+c_{\delta} .
$$

We have now to handle the last integral on the right-hand side of (4.20). To this aim, we observe that Lemma 4.4 and Young's inequality entail

$$
\begin{aligned}
& \delta\left|\beta_{\varepsilon}\left(u_{\varepsilon}\right)\right|^{2}-\beta_{\varepsilon}\left(u_{\varepsilon}\right) \beta_{\Gamma, \varepsilon}\left(u_{\varepsilon}\right)=\delta\left|\beta_{\varepsilon}\left(u_{\varepsilon}\right)\right|^{2}-\left|\beta_{\varepsilon}\left(u_{\varepsilon}\right)\right|\left|\beta_{\Gamma, \varepsilon}\left(u_{\varepsilon}\right)\right| \\
& \leq\left|\beta_{\varepsilon}\left(u_{\varepsilon}\right)\right|^{2}(\delta-1 / \eta)+C_{\Gamma}\left|\beta_{\varepsilon}\left(u_{\varepsilon}\right)\right| / \eta \\
& \leq\left|\beta_{\varepsilon}\left(u_{\varepsilon}\right)\right|^{2}(\delta-1 / 2 \eta)+C_{\Gamma}^{2} / 2 \eta \leq C_{\Gamma}^{2} / 2 \eta \quad \text { a.e. on } \Sigma
\end{aligned}
$$

whenever $\delta<1 / 2 \eta$. Thus, by fixing $\delta<1 / 2 \eta$ it turns out that the last integral of (4.20) is uniformly bounded. Then, neglecting the first four positive terms on the left-hand side, from (4.20) it follows that

$$
\left\|\beta_{\varepsilon}\left(u_{\varepsilon}\right)\right\|_{L^{2}(0, T ; H)}+\left\|\beta_{\varepsilon}\left(u_{\varepsilon}\right)\right\|_{L^{2}\left(0, T ; H_{\Gamma}\right)} \leq C .
$$

The first part of estimate (4.21) has some consequences on the regularity of $u_{\varepsilon}$ and of the boundary term $\partial_{n} u_{\varepsilon}$. Indeed, taking an arbitrary $z \in H_{0}^{1}(\Omega)$ as test function in (4.8), we can recover the partial differential equation satisfied by $u_{\varepsilon}$ in $Q$, which can be written as

$$
-\Delta u_{\varepsilon}=f-\partial_{t} u_{\varepsilon}-\beta_{\varepsilon}\left(u_{\varepsilon}\right)-\pi\left(u_{\varepsilon}\right)
$$

and holds (for instance) in the sense of distributions on $Q$. Now, thanks to the estimates (4.13) and (4.21) and in view of the assumption (2.12) on $f$ and of the Lipschitz continuity of $\pi$, by comparison in (4.22) we deduce that

$$
\left\|-\Delta u_{\varepsilon}\right\|_{L^{2}(0, T ; H)} \leq C .
$$


Hence, recalling (4.13) and using e.g. [5, Theorem 3.1, p. 1.79], applied in this case with

$$
A=-\Delta, \quad g_{0}=\left.u_{\varepsilon}\right|_{\Gamma}, \quad p=2, \quad r=0, \quad t=1, \quad s=\frac{3}{2},
$$

we deduce the estimate

$$
\int_{0}^{T}\left\|u_{\varepsilon}(t)\right\|_{H^{3 / 2}(\Omega)}^{2} d t \leq c \int_{0}^{T}\left(\left\|\Delta u_{\varepsilon}(t)\right\|_{H}^{2}+\left\|u_{\varepsilon}(t)\right\|_{V_{\Gamma}}^{2}\right) d t
$$

which implies (cf. (4.13)

$$
\nu^{1 / 2}\left\|u_{\varepsilon}\right\|_{L^{2}\left(0, T: H^{3 / 2}(\Omega)\right)} \leq C .
$$

By virtue of (4.23), (4.25) and [5, Theorem 2.27, p. 1.64] we are also able to conclude that

$$
\nu^{1 / 2}\left\|\partial_{n} u_{\varepsilon}\right\|_{L^{2}\left(0, T ; H_{\Gamma}\right)} \leq C
$$

\subsubsection{Fourth a priori estimate}

Using now (4.22) and (4.26), we are able to recover from (4.8) the variational formulation of the boundary equation

$$
\partial_{n} u_{\varepsilon}+\partial_{t} u_{\varepsilon}-\nu \Delta_{\Gamma} u_{\varepsilon}+\beta_{\Gamma, \varepsilon}\left(u_{\varepsilon}\right)+\pi_{\Gamma}\left(u_{\varepsilon}\right)=f_{\Gamma}
$$

on $\Sigma$, that is

$$
\int_{\Gamma} \partial_{n} u_{\varepsilon} z+\int_{\Gamma} \partial_{t} u_{\varepsilon} z+\nu \int_{\Gamma} \nabla_{\Gamma} u_{\varepsilon} \cdot \nabla_{\Gamma} z+\int_{\Gamma} \beta_{\Gamma, \varepsilon}\left(u_{\varepsilon}\right) z+\int_{\Gamma} \pi_{\Gamma}\left(u_{\varepsilon}\right) z=\int_{\Gamma} f_{\Gamma} z
$$

for every $z \in \mathcal{V}$, a.e. in $(0, T)$. We aim to find an estimate for $\beta_{\Gamma, \varepsilon}\left(u_{\varepsilon}\right)$. Then, we test the above equation by $\beta_{\Gamma, \varepsilon}\left(u_{\varepsilon}\right)$ and integrate over $(0, T)$. We obtain

$$
\begin{aligned}
& \int_{\Gamma} \hat{\beta}_{\Gamma, \varepsilon}\left(u_{\varepsilon}(T)\right)+\nu \int_{\Sigma} \beta_{\Gamma, \varepsilon}^{\prime}\left(u_{\varepsilon}\right)\left|\nabla_{\Gamma} u_{\varepsilon}\right|^{2}+\int_{\Sigma}\left|\beta_{\Gamma, \varepsilon}\left(u_{\varepsilon}\right)\right|^{2} \\
& =\int_{\Gamma} \hat{\beta}_{\Gamma, \varepsilon}\left(u_{0}\right)+\int_{\Sigma}\left(f_{\Gamma}-\pi_{\Gamma}\left(u_{\varepsilon}\right)-\partial_{n} u_{\varepsilon}\right) \beta_{\Gamma, \varepsilon}\left(u_{\varepsilon}\right) .
\end{aligned}
$$

The first integral on the right-hand side of (4.28) is bounded, thanks to (4.4) and (2.21). In fact, we have

$$
\int_{\Gamma} \hat{\beta}_{\Gamma, \varepsilon}\left(u_{0}\right) \leq\left\|\hat{\beta}_{\Gamma}\left(u_{0}\right)\right\|_{L^{1}(\Gamma)} .
$$

For the last integral on the right-hand side we invoke (2.12), (2.2), (4.11) and (4.26): then Young's inequality helps us to infer that

$$
\int_{\Sigma}\left(f_{\Gamma}-\pi_{\Gamma}\left(u_{\varepsilon}\right)-\partial_{n} u_{\varepsilon}\right) \beta_{\Gamma, \varepsilon}\left(u_{\varepsilon}\right) \leq \frac{1}{2} \int_{\Sigma}\left|\beta_{\Gamma, \varepsilon}\left(u_{\varepsilon}\right)\right|^{2}+C\left(1+\nu^{-1}\right) .
$$

Therefore, forgetting the two positive integrals on the left-hand side of (4.28), we plainly obtain

$$
\nu^{1 / 2}\left\|\beta_{\Gamma, \varepsilon}\left(u_{\varepsilon}\right)\right\|_{L^{2}\left(0, T ; H_{\Gamma}\right)} \leq C .
$$




\subsection{Passage to the limit}

Thanks to the previous estimates, we can now prove the existence of the solution, thus completing the proof of Theorem 2.5. Owing to (4.13), (4.21) and (4.29) and by standard compactness results, we infer that limit functions exist such that the following convergences hold true:

$$
\begin{aligned}
u_{\varepsilon} \stackrel{*}{\rightarrow} u & \text { in } L^{\infty}(0, T, V) \cap H^{1}(0, T ; H), \\
\left.\left.u_{\varepsilon}\right|_{\Gamma} \stackrel{*}{*} u\right|_{\Gamma}=v & \text { in } L^{\infty}\left(0, T ; V_{\Gamma}\right) \cap H^{1}\left(0, T ; H_{\Gamma}\right), \\
\beta_{\varepsilon}\left(u_{\varepsilon}\right) \rightarrow \xi & \text { in } L^{2}(0, T ; H), \\
\beta_{\Gamma, \varepsilon}\left(\left.u_{\varepsilon}\right|_{\Gamma}\right) \rightarrow \xi_{\Gamma} & \text { in } L^{2}\left(0, T ; H_{\Gamma}\right)
\end{aligned}
$$

at least for a not relabeled subsequence, as $\varepsilon \searrow 0$.

Now, we want to prove that the quadruplet $\left(u, v, \xi, \xi_{\Gamma}\right)$ is a solution to our problem (2.13)-(2.18). Recalling that the embeddings $V \subset H$ and $V_{\Gamma} \subset H_{\Gamma}$ are compact, we can apply the result in [21, Sect. 8, Cor. 4] to infer that

$$
\begin{aligned}
u_{\varepsilon} \rightarrow u & \text { strongly in } C^{0}([0, T] ; H), \\
\left.u_{\varepsilon}\right|_{\Gamma} \rightarrow v & \text { strongly in } C^{0}\left([0, T] ; H_{\Gamma}\right) .
\end{aligned}
$$

Note that the initial conditions (2.17) are fulfilled and moreover, because of the Lipschitz continuity of $\pi$ and $\pi_{\Gamma}$, we have that

$$
\begin{aligned}
\pi\left(u_{\varepsilon}\right) \rightarrow \pi(u) & \text { strongly in } C^{0}([0, T] ; H), \\
\pi_{\Gamma}\left(\left.u_{\varepsilon}\right|_{\Gamma}\right) \rightarrow \pi_{\Gamma}(v) & \text { strongly in } C^{0}\left([0, T] ; H_{\Gamma}\right) .
\end{aligned}
$$

Then, passing to the limit in (4.8), we recover the variational equality (2.18). It remains to check (2.15)-(2.16). Since (4.32) holds and $u_{\varepsilon} \rightarrow u$ strongly in $L^{2}(0, T ; H)$, we easily deduce

$$
\limsup _{\varepsilon \searrow 0} \int_{Q} \beta_{\varepsilon}\left(u_{\varepsilon}\right) u_{\varepsilon}=\int_{Q} \xi u,
$$

which allows us to apply [2, Prop. 1.1, Ch. II] and conclude that $\xi \in \beta(u)$ a.e. in $Q$. We can argue in a similar way for $\beta_{\Gamma, \varepsilon}$ and obtain $\xi_{\Gamma} \in \beta_{\Gamma}(v)$ a.e. on $\Sigma$. Therefore, the proof of Theorem 2.5 is complete.

Remark 4.5 (Further regularities). In our passage to the limit we can recover further regularities for the limit function $u$. In fact, from (4.23) we infer that $\Delta u$ belongs to $L^{2}(0, T ; H)$ just by the lower semicontinuity property of the norm. Similarly, we deduce from (4.26) that $\partial_{n} u$ is in $L^{2}\left(0, T ; H_{\Gamma}\right)$. Now, since $L^{2}\left(0, T ; H_{\Gamma}\right) \subset L^{2}\left(0, T ; H^{-1 / 4}(\Gamma)\right)$, we can consider $\partial_{n} u$ as an element of $L^{2}\left(0, T ; H^{-1 / 4}(\Gamma)\right)$ and then read the equation (2.7) as the elliptic equation

$$
-\Delta_{\Gamma} v+v=F_{\Gamma}
$$

where

$$
F_{\Gamma}:=\frac{1}{\nu}\left(f_{\Gamma}-\partial_{n} u-\partial_{t} v-\xi_{\Gamma}-\pi_{\Gamma}(v)\right)+v \in L^{2}\left(0, T ; H^{-1 / 4}(\Gamma)\right)
$$

Using now the boundary version of [18, Theorem 7.5, p. 204], we can deduce that $v=\left.u\right|_{\Gamma}$ belongs to $L^{2}\left(0, T ; H^{2-1 / 4}(\Gamma)\right) \subset L^{2}\left(0, T ; H^{3 / 2}(\Gamma)\right)$, whence also

$$
u \in L^{2}\left(0, T ; H^{2}(\Omega)\right)
$$


thanks to [5, Theorem 3.1, p. 1.79]. Moreover, we deduce that

$$
\partial_{n} u \in L^{2}\left(0, T ; H^{1 / 2}(\Gamma)\right) .
$$

\section{The case without the Laplace-Beltrami operator}

In this section we deal with the case in which the parameter $\nu$ of the problem, which so far has been assumed to be positive, is equal to 0 . Both the operators $\Delta_{\Gamma}$ and $\nabla_{\Gamma}$ formally disappear and then the corresponding contributions have to be ignored. In particular, the case $\nu=0$ is significant in one space dimension since the above boundary operators are meaningless in this case. Before stating our main results, we have to redefine the operator induced on $H_{\Gamma}$ by $\beta_{\Gamma}$, extending it in a suitable way.

\subsection{Extension of the subdifferential on the boundary}

We introduce now another boundary space

$$
W_{\Gamma}:=H^{1 / 2}(\Gamma)
$$

in place of $V_{\Gamma}$ and note that, consequently, the new space of the test functions (replacing $\mathcal{V}$ ) is nothing but $V$, thanks to the trace theorem. Recalling the identification $H_{\Gamma} \cong H_{\Gamma}^{*}$, let us indicate by $W_{\Gamma}^{*}$ the dual space of $W_{\Gamma}$, that is the space $W_{\Gamma}^{*}:=H^{-1 / 2}(\Gamma)$.

In view of the definition (2.1) of the function $\hat{\beta}_{\Gamma}$, we introduce a suitable generalization of the induced operator $\beta_{\Gamma}$ acting on the boundary. To this aim, we associate to the function $\hat{\beta}_{\Gamma}$ the functionals $\hat{\beta}_{\Gamma_{H_{\Gamma}}}(z)$ on $H_{\Gamma}$ and $\hat{\beta}_{\Gamma_{W_{\Gamma}}}(z)$ on $W_{\Gamma}$ defined by

$$
\begin{aligned}
& \hat{\beta}_{\Gamma_{H_{\Gamma}}}(z):=\left\{\begin{array}{ll}
\int_{\Gamma} \hat{\beta}_{\Gamma}(z) & \text { if } z \in H_{\Gamma} \text { and } \hat{\beta}_{\Gamma}(z) \in L^{1}(\Gamma) \\
+\infty & \text { if } z \in H_{\Gamma} \text { and } \hat{\beta}_{\Gamma}(z) \notin L^{1}(\Gamma)
\end{array},\right. \\
& \hat{\beta}_{\Gamma_{W_{\Gamma}}}(z):=\hat{\beta}_{\Gamma_{H_{\Gamma}}}(z) \text { if } z \in W_{\Gamma} .
\end{aligned}
$$

As it is well-known, $\hat{\beta}_{\Gamma_{H_{\Gamma}}}$ and $\hat{\beta}_{\Gamma_{W_{\Gamma}}}$ are convex and lower semicontinuous functionals on $H_{\Gamma}$ and $W_{\Gamma}$, respectively. They are also proper since $W_{\Gamma}$ contains all the constant functions. Now, we denote by $\beta_{\Gamma_{W_{\Gamma}^{*}}^{*}}:=\partial \hat{\beta}_{\Gamma_{W_{\Gamma}}}: W_{\Gamma} \rightarrow 2^{W_{\Gamma}^{*}}$ the subdifferential of $\hat{\beta}_{\Gamma_{W_{\Gamma}}}$, which is defined by

$$
\begin{array}{r}
\rho \in \beta_{\Gamma_{W_{\Gamma}^{*}}}(z) \text { if and only if } \rho \in W_{\Gamma}^{*}, z \in D\left(\hat{\beta}_{\Gamma_{W_{\Gamma}}}\right) \text { and } \\
\hat{\beta}_{\Gamma_{W_{\Gamma}}}(z) \leq W_{\Gamma}^{*}\langle\rho, z-w\rangle_{W_{\Gamma}}+\hat{\beta}_{\Gamma_{W_{\Gamma}}}(w) \quad \forall w \in W_{\Gamma} .
\end{array}
$$

This turns out to be a maximal monotone operator from $W_{\Gamma}$ to $W_{\Gamma}^{*}$ satisfying $\beta_{\Gamma_{W_{\Gamma}^{*}}}(0) \ni$ 0 (cf. (2.1) ). We observe that such an operator is strictly related to the operator $\beta_{\Gamma}$ employed in previous sections since (see, e.g., [3. Prop. 2.5]) $\rho \in \beta_{\Gamma}(z)$ in $H_{\Gamma}$ if and only if $\rho \in \beta_{\Gamma_{W_{\Gamma}^{*}}}(z)$ and $\rho \in H_{\Gamma}$. We emphasize that in the framework $\nu=0$ we are no longer able to deal with the natural extension to $H_{\Gamma}$ of $\beta_{\Gamma}$. The inclusion (2.16) governing the 
dynamic of the phase is in fact reinterpreted by (5.1) in the abstract setting of the duality pairing between $W_{\Gamma}^{*}$ and $W_{\Gamma}$. Nevertheless, the physical consistence is somehow preserved since such an inclusion forces the phase to assume only meaningful values in the domain of $\beta_{\Gamma_{W_{\Gamma}^{*}}}$; further, if $\xi_{\Gamma}(t) \in \beta_{\Gamma_{W_{\Gamma}^{*}}}(v(t))$ for a.e. $t \in(0, T)$, we have in particular that $v(t) \in D\left(\hat{\beta}_{\Gamma}\right)$ a.e. on $\Gamma$.

\subsection{Properties of the solution}

Let us formulate the new problem. As we have done before, we ask the data to fulfill at first the general assumptions (2.12) and we look now for a quadruplet $\left(u, v, \xi, \xi_{\Gamma}\right)$ such that

$$
\begin{aligned}
& u \in L^{2}(0, T ; V) \cap H^{1}\left(0, T ; V^{*}\right), \\
& v:=\left.u\right|_{\Gamma} \in L^{2}\left(0, T ; W_{\Gamma}\right) \cap H^{1}\left(0, T ; W_{\Gamma}^{*}\right), \\
& \xi \in L^{2}(0, T ; H) \text { and } \xi \in \beta(u) \text { a.e. in } Q, \\
& \xi_{\Gamma} \in L^{2}\left(0, T ; W_{\Gamma}^{*}\right) \text { and } \xi_{\Gamma}(t) \in \beta_{\Gamma_{W_{\Gamma}^{*}}}(v(t)) \text { for a.e. } t \in(0, T), \\
& u(0)=u_{0}, \quad v(0)=v_{0}
\end{aligned}
$$

and satisfying for a.a. $t \in(0, T)$ and every $z \in V$

$$
\begin{aligned}
\left\langle\partial_{t} u(t), z\right\rangle+ & \int_{\Omega} \nabla u(t) \cdot \nabla z+\int_{\Omega}(\xi(t)+\pi(u(t))) z+W_{\Gamma}^{*}\left\langle\partial_{t} v(t), z\right\rangle_{W_{\Gamma}} \\
& +\int_{\Gamma} \pi_{\Gamma}(v(t)) z+{ }_{W_{\Gamma}^{*}}\left\langle\xi_{\Gamma}(t), z\right\rangle_{W_{\Gamma}}=\int_{\Omega} f(t) z+\int_{\Gamma} f_{\Gamma}(t) z .
\end{aligned}
$$

We state now the modified result of continuous dependence on the data and uniqueness: we observe that this is very similar to the one given for the case $\nu>0$, the only difference being the disappearance of the term with the factor $\nu$. We do not present the proof of the theorem because it follows faithfully the arguments developed in Section 3 ,

Theorem 5.1 (Continuous dependence and uniqueness). Under the assumptions (2.1) (2.4), (2.12), (5.1), let $\left(u_{1}, v_{1}\right)$ and $\left(u_{2}, v_{2}\right)$ be the first two components of two different solutions of the problem (5.2) -(5.7), each one corresponding to a set of data $u_{0, i}, v_{0, i}, f_{i}, f_{\Gamma, i}$ with $i=1,2$. Then, there is a constant $c$ such that

$$
\begin{aligned}
& \left\|u_{1}-u_{2}\right\|_{L^{\infty}(0, T ; H)}^{2}+\left\|\nabla\left(u_{1}-u_{2}\right)\right\|_{L^{2}(Q)}^{2}+\left\|v_{1}-v_{2}\right\|_{L^{\infty}\left(0, T ; H_{\Gamma}\right)}^{2} \\
& \leq c\left\{\left\|u_{0,1}-u_{0,2}\right\|_{H}^{2}+\left\|v_{0,1}-v_{0,2}\right\|_{H_{\Gamma}}^{2}+\left\|f_{1}-f_{2}\right\|_{L^{2}(0, T ; H)}^{2}+\left\|f_{\Gamma, 1}-f_{\Gamma, 2}\right\|_{L^{2}\left(0, T ; H_{\Gamma}\right)}^{2}\right\} .
\end{aligned}
$$

Moreover, any two solutions of the problem (5.2)-(5.7) with the same data necessarily coincide.

In the following we denote by $\left(u^{\nu}, v^{\nu}, \xi^{\nu}, \xi_{\Gamma}^{\nu}\right)$ the solution of the problem in the case $\nu>0$ (provided by Theorems 2.5 and 2.4) and by $\left(u, v, \xi, \xi_{\Gamma}\right)$ the expected solution in the case $\nu=0$. Even in this case we have to reinforce our assumptions on the data requiring:

$$
\begin{aligned}
& u_{0} \in V, \quad \text { so that } v_{0}=\left.u_{0}\right|_{\Gamma} \in W_{\Gamma}, \\
& \hat{\beta}\left(u_{0}\right) \in L^{1}(\Omega) \text { and } \hat{\beta}_{\Gamma}\left(v_{0}\right) \in L^{1}(\Gamma) .
\end{aligned}
$$


Note that (5.8) is weaker than (2.20) (cf. (2.10) ). Since we want to prove that the solution $\left(u, v, \xi, \xi_{\Gamma}\right)$ can be obtained as the asymptotic limit of the quadruplet $\left(u^{\nu}, v^{\nu}, \xi^{\nu}, \xi_{\Gamma}^{\nu}\right)$, we have to approximate the initial data $u_{0}, v_{0}$ in order to comply with the regularity in (2.20)-(2.21). Then, we introduce a sequence $\left\{u_{0 \nu}\right\}$ such that

$$
\begin{aligned}
& u_{0 \nu} \in V \text { and } v_{0 \nu}=\left.u_{0 \nu}\right|_{\Gamma} \in V_{\Gamma}, \\
& u_{0 \nu} \rightarrow u_{0} \text { in } V, \text { whence } v_{0 \nu} \rightarrow v_{0} \text { in } W_{\Gamma} \text { as } \nu \searrow 0, \\
& \nu^{1 / 2}\left\|v_{0 \nu}\right\|_{V_{\Gamma}}^{2}+\int_{\Omega} \hat{\beta}\left(u_{0 \nu}\right)+\int_{\Gamma} \hat{\beta}_{\Gamma}\left(v_{0 \nu}\right) \leq C .
\end{aligned}
$$

Now, the question for the reader is whether such a sequence $\left\{u_{0 \nu}\right\}$ exists. Of course, if $v_{0} \in V_{\Gamma}$ then we can take $u_{0 \nu}=u_{0}$ for all $\nu>0$. However, the answer is positive in general.

Lemma 5.2. Under the assumptions (5.8)-(5.9), there is a sequence $\left\{u_{0 \nu}\right\}$ such that $u_{0 \nu}$ and $v_{0 \nu}=\left.u_{0 \nu}\right|_{\Gamma}$ satisfy (5.10) $-(\underline{5.12})$.

Proof. One can choose $u_{0 \nu}$ as the solution of the following variational equality

$$
\int_{\Omega}\left(u_{0 \nu}-u_{0}\right) z+\nu \int_{\Omega} \nabla u_{0 \nu} \cdot \nabla z+\nu \int_{\Gamma} \rho_{0 \nu} z=0 \quad \text { for all } z \in V,
$$

where

$$
\rho_{0 \nu} \in \beta_{\Gamma}\left(v_{0 \nu}\right) \text { a.e. in } \Gamma \text {. }
$$

Indeed, consider the function

$$
\Phi(z)= \begin{cases}\frac{1}{2} \int_{\Omega}|\nabla z|^{2}+\int_{\Gamma} \hat{\beta}_{\Gamma}\left(\left.z\right|_{\Gamma}\right) & \text { if } z \in V \text { and } \hat{\beta}_{\Gamma}\left(\left.z\right|_{\Gamma}\right) \in L^{1}(\Gamma) \\ +\infty & \text { otherwise }\end{cases}
$$

which is proper, convex and lower semicontinuos in $H$. The subdifferential of $\Phi$ is a maximal monotone operator from $H$ to $H$ (see [2, Example 4, pp. 63-67]) with domain

$$
\left\{z \in H^{2}(\Omega):-\partial_{n} z \in \beta_{\Gamma}\left(\left.z\right|_{\Gamma}\right) \text { a.e. in } \Gamma\right\} .
$$

Then, it is straightforward to deduce the existence and uniqueness of $u_{0 \nu} \in H^{2}(\Omega)$ solving (5.13)-(5.14). Moreover, $u_{0 \nu}$ turns out to be the solution to the boundary value problem

$$
\begin{aligned}
& u_{0 \nu}-\nu \Delta u_{0 \nu}=u_{0} \text { in } \Omega, \\
& \partial_{n} u_{0 \nu}+\beta_{\Gamma}\left(v_{0 \nu}\right) \ni 0 \text { on } \Gamma .
\end{aligned}
$$

Now, taking $z=u_{0 \nu}-u_{0}$ in (5.13), we easily recover the estimate

$$
\frac{1}{\nu}\left\|u_{0 \nu}-u_{0}\right\|_{H}^{2}+\Phi\left(u_{0 \nu}\right) \leq \Phi\left(u_{0}\right),
$$

whence (5.10)-(5.11) and part of (5.12), namely

$$
\int_{\Gamma} \hat{\beta}_{\Gamma}\left(v_{0 \nu}\right) \leq C
$$


follow. To complete the verification of (5.12), we can take $z=\beta_{\varepsilon}\left(u_{0 \nu}\right)$ in (5.13) , recalling that $\beta_{\varepsilon}$ denotes the Yosida regularization of $\beta$. We obtain

$$
\int_{\Omega} \hat{\beta}_{\varepsilon}\left(u_{0 \nu}\right)+\nu \int_{\Omega} \beta_{\varepsilon}^{\prime}\left(u_{0 \nu}\right)\left|\nabla u_{0 \nu}\right|^{2}+\nu \int_{\Gamma} \rho_{0 \nu} \beta_{\varepsilon}\left(v_{0 \nu}\right) \leq \int_{\Omega} \hat{\beta}_{\varepsilon}\left(u_{0}\right)
$$

and, observing that the second and third terms on the left-hand side are nonnegative (see (5.14) and note that $0 \in \beta_{\Gamma}(0)$ and $\left.\beta_{\varepsilon}(0)=0\right)$, by (4.4) and [4, Prop. 2.11, p. 39] we have

$$
\int_{\Omega} \hat{\beta}_{\varepsilon}\left(u_{0 \nu}\right) \leq \int_{\Omega} \hat{\beta}\left(u_{0}\right) \text { for all } \varepsilon \in(0,1) \text {, so that } \int_{\Omega} \hat{\beta}\left(u_{0 \nu}\right) \leq C \text {. }
$$

Finally, using the estimate (2.23) in [2, p. 64], we find out that

$$
\left\|u_{0 \nu}\right\|_{H^{2}(\Omega)} \leq C\left(1+\left\|u_{0 \nu}\right\|_{H}+\nu^{-1}\left\|u_{0 \nu}-u_{0}\right\|_{H}\right) \leq C\left(1+\nu^{-1 / 2}\right)
$$

thanks to (5.17). Then, it turns out that $\nu^{1 / 2}\left\|v_{0 \nu}\right\|_{H^{3 / 2}(\Gamma)} \leq C$ and property (5.12) is ensured.

Next theorem shows the existence of the solution to the problem (5.2)-(5.7) along with suitable convergences of the components $u^{\nu}, v^{\nu}, \xi^{\nu}, \xi_{\Gamma}^{\nu}$ of the solution to the problem with $\nu>0$ to the respective components $u, v, \xi, \xi_{\Gamma}$.

Theorem 5.3 (Existence and convergence as $\nu \searrow 0$ ). Assume (2.1) -(2.4), (2.22)-(2.23), (2.12), (5.1) and (5.8)-(5.9). Then there exists a quadruplet $\left(u, v, \xi, \xi_{\Gamma}\right)$ satisfying

$$
\begin{aligned}
& u \in L^{\infty}(0, T ; V) \cap H^{1}(0, T ; H), \\
& v \in L^{\infty}\left(0, T ; W_{\Gamma}\right) \cap H^{1}\left(0, T ; H_{\Gamma}\right)
\end{aligned}
$$

and solving problem (5.2)-(5.7). Moreover, denoting by $\left(u^{\nu}, v^{\nu}, \xi^{\nu}, \xi_{\Gamma}^{\nu}\right)$ the solution to the problem (2.13)-(2.18) with initial data $u_{0 \nu}, v_{0 \nu}$ as in (5.10)-(5.12), the following convergences hold as $\nu \searrow 0$ :

$$
\begin{aligned}
u^{\nu} \stackrel{*}{\rightarrow} u & \text { in } L^{\infty}(0, T ; V) \cap H^{1}(0, T ; H), \\
v^{\nu} \stackrel{*}{\rightarrow} v & \text { in } L^{\infty}\left(0, T ; W_{\Gamma}\right) \cap H^{1}\left(0, T ; H_{\Gamma}\right), \\
\xi^{\nu} \rightarrow \xi & \text { in } L^{2}(0, T ; H), \\
\xi_{\Gamma}^{\nu} \rightarrow \xi_{\Gamma} & \text { in } L^{2}\left(0, T, W_{\Gamma}^{*}\right) .
\end{aligned}
$$

Proof. We prove the convergences (5.20)-(5.23) by using compactness arguments. Let us recall the a priori estimates (4.11), (4.13), (4.21) and (4.23) holding for the solution $\left(u^{\nu}, v^{\nu}, \xi^{\nu}, \xi_{\Gamma}^{\nu}\right)$. In particular, let us point out that

$$
\begin{aligned}
\left\|u^{\nu}\right\|_{L^{\infty}(0, T ; V) \cap H^{1}(0, T ; H)}+\left\|v^{\nu}\right\|_{L^{\infty}\left(0, T ; W_{\Gamma}\right) \cap H^{1}\left(0, T ; H_{\Gamma}\right)} \\
+\nu^{1 / 2}\left\|v^{\nu}\right\|_{L^{\infty}\left(0, T ; V_{\Gamma}\right)}+\left\|\xi^{\nu}\right\|_{L^{2}(0, T ; H)} \leq C .
\end{aligned}
$$

In view of (4.26) and (4.29), it is clear that we cannot deduce a uniform bound for $\partial_{n} u^{\nu}$ and $\xi_{\Gamma}^{\nu}$ in $L^{2}\left(0, T ; H_{\Gamma}\right)$ independently of $\nu$. On the other hand, with a similar reasoning and taking advantage of (4.23), we can apply once more [5, Theorem 3.1, p. 1.79] and [5, 
Theorem 2.27, p. 1.64], where the new choice of operators and parameters is now given by

$$
A=-\Delta, \quad g_{0}=\left.u^{\nu}\right|_{\Gamma}, \quad p=2, \quad r=0, \quad t=\frac{1}{2}, \quad s=1 .
$$

Thus, we find the following estimate for the normal derivative

$$
\left\|\partial_{n} u^{\nu}\right\|_{L^{2}\left(0, T ; W_{\Gamma}^{*}\right)} \leq C
$$

Next, let us consider the analog of (4.27), i.e.,

$$
\partial_{n} u^{\nu}+\partial_{t} u^{\nu}-\nu \Delta_{\Gamma} u^{\nu}+\xi_{\Gamma}^{\nu}+\pi_{\Gamma}\left(u^{\nu}\right)=f_{\Gamma} \quad \text { in } L^{2}\left(0, T ; H_{\Gamma}\right),
$$

which holds in $W_{\Gamma}^{*}$, a.e. in $(0, T)$ as well. Now, owing to (4.26) and (4.29), multiplying (5.26) by $\nu^{1 / 2}$, from a comparison of terms we obtain

$$
\nu^{3 / 2}\left\|\Delta_{\Gamma} u^{\nu}\right\|_{L^{2}\left(0, T ; H_{\Gamma}\right)} \leq C
$$

Due to (4.11) and to the fact that $\Delta_{\Gamma}$ is a linear and bounded operator from $V_{\Gamma}$ to $V_{\Gamma}^{*}\left(\cong H^{-1}(\Gamma)\right)$, we have

$$
\nu^{1 / 2}\left\|\Delta_{\Gamma} u^{\nu}\right\|_{L^{2}\left(0, T ; V_{\Gamma}^{*}\right)} \leq C .
$$

Then, combining (5.27) and (5.28), by interpolation (see, e.g., [5, Theorem 2.20, p. 1.53]) we deduce that

$$
\nu\left\|\Delta_{\Gamma} u^{\nu}\right\|_{L^{2}\left(0, T ; W_{\Gamma}^{*}\right)} \leq C .
$$

Therefore, thanks to (5.25) and (5.29), a further comparison in (5.26) leads to

$$
\left\|\xi_{\Gamma}^{\nu}\right\|_{L^{2}\left(0, T ; W_{\Gamma}^{*}\right)} \leq C .
$$

Collecting then (5.24) and (5.30), we conclude that there exists a quadruplet $\left(u, v, \xi, \xi_{\Gamma}\right)$ such that the convergences (5.20)-(5.23) and

$$
\nu v^{\nu} \rightarrow 0 \quad \text { in } L^{\infty}\left(0, T ; V_{\Gamma}\right)
$$

hold as $\nu \searrow 0$, in principle for a subsequence and then for the whole family once we have checked that $\left(u, v, \xi, \xi_{\Gamma}\right)$ is the unique solution to the problem (5.2)-(5.7).

In order to verify that the limit quadruplet $\left(u, v, \xi, \xi_{\Gamma}\right)$ solves problem (5.2)-(5.7), we can proceed as in Subsection 4.2. In particular, we note that, as in (4.34)-(4.35), we still have

$$
\begin{array}{cc}
u^{\nu} \rightarrow u & \text { strongly in } C^{0}([0, T] ; H), \\
v^{\nu} \rightarrow v & \text { strongly in } C^{0}\left([0, T] ; H_{\Gamma}\right),
\end{array}
$$

as the embedding $W_{\Gamma} \subset H_{\Gamma}$ is also compact. Then, it is straightforward to obtain (5.7) when passing to the limit in (2.18); initial conditions (5.6) follow from (5.32)-(5.33) and 
(5.11). Moreover, (5.4) can be deduced from, e.g., [4, Prop. 2.5, p. 27] thanks to (2.16), (5.22), (5.32), and

$$
\limsup _{\nu \searrow 0} \int_{Q} \xi^{\nu} u^{\nu} \leq \int_{Q} \xi u
$$

We point out that (5.34) implies not only (5.4) but the additional property

$$
\lim _{\nu \searrow 0} \int_{Q} \xi^{\nu} u^{\nu}=\int_{Q} \xi u
$$

Analogously, one should prove that

$$
\limsup _{\nu \searrow 0} \int_{0}^{T} W_{\Gamma}^{*}\left\langle\xi_{\Gamma}^{\nu}(t), v^{\nu}(t)\right\rangle_{W_{\Gamma}} d t \leq \int_{0}^{T} W_{\Gamma}^{*}\left\langle\xi_{\Gamma}(t), v(t)\right\rangle_{W_{\Gamma}} d t,
$$

in order to infer (5.5). From (2.16) it follows that (5.1) holds for $\rho=\xi_{\Gamma}^{\nu}$ a.e. in $(0, T)$, whence

$$
\int_{\Sigma} \xi_{\Gamma}^{\nu} v^{\nu}=\int_{0}^{T} W_{\Gamma}^{*}\left\langle\xi_{\Gamma}^{\nu}(t), v^{\nu}(t)\right\rangle_{W_{\Gamma}} d t
$$

Moreover, the convergences (5.23) and (5.21) are in force. Then, taking $z=u^{\nu}(t)$ in (2.18) and integrating with respect to $t$, we obtain

$$
\begin{gathered}
\int_{0}^{T} W_{\Gamma}^{*}\left\langle\xi_{\Gamma}^{\nu}(t), v^{\nu}(t)\right\rangle_{W_{\Gamma}} d t=\frac{1}{2}\left\|u_{0 \nu}\right\|_{H}^{2}-\frac{1}{2}\left\|u^{\nu}(T)\right\|_{H}^{2}-\left\|\nabla u^{\nu}\right\|_{L^{2}(0, T ; H)}^{2}+\int_{Q}\left(f-\pi\left(u^{\nu}\right)\right) u^{\nu} \\
-\int_{Q} \xi^{\nu} u^{\nu}+\frac{1}{2}\left\|v_{0 \nu}\right\|_{H_{\Gamma}}^{2}-\frac{1}{2}\left\|v^{\nu}(T)\right\|_{H_{\Gamma}}^{2}-\nu\left\|\nabla_{\Gamma} v^{\nu}\right\|_{L^{2}\left(0, T ; H_{\Gamma}\right)}^{2}+\int_{\Sigma}\left(f_{\Gamma}-\pi_{\Gamma}\left(v^{\nu}\right)\right) v^{\nu} .
\end{gathered}
$$

We pass now to the limsup as $\nu \searrow 0$, using (5.11), (5.32)-(5.33), (5.20) and the lower semicontinuity of the functional $z \mapsto\|\nabla z\|_{L^{2}(0, T ; H)}^{2}$ in the weak star topology of $L^{\infty}(0, T ; V)$, the Lipschitz continuity of $\pi$ and $\pi_{\Gamma},(5.35)$ and the fact that

$$
-\nu\left\|\nabla_{\Gamma} v^{\nu}\right\|_{L^{2}\left(0, T ; H_{\Gamma}\right)}^{2} \leq 0
$$

Thus, it is straightforward to infer the inequality

$$
\begin{aligned}
\underset{\nu \searrow 0}{\limsup } & \int_{0}^{T} W_{\Gamma}^{*}\left\langle\xi_{\Gamma}^{\nu}(t), v^{\nu}(t)\right\rangle_{W_{\Gamma}} d t \leq \frac{1}{2}\left\|u_{0}\right\|_{H}^{2}-\frac{1}{2}\|u(T)\|_{H}^{2}-\|\nabla u\|_{L^{2}(0, T ; H)}^{2} \\
& \int_{Q}(f-\pi(u)-\xi) u+\frac{1}{2}\left\|v_{0}\right\|_{H_{\Gamma}}^{2}-\frac{1}{2}\|v(T)\|_{H_{\Gamma}}^{2}+\int_{\Sigma}\left(f_{\Gamma}-\pi_{\Gamma}(v)\right) v
\end{aligned}
$$

and see that the right-hand side is nothing but

$$
\int_{0}^{T} W_{\Gamma}^{*}\left\langle\xi_{\Gamma}(t), v(t)\right\rangle_{W_{\Gamma}} d t
$$

thanks to (5.7) and (5.6). Hence (5.36) follows and Theorem 5.3 is completely proved. 


\section{Acknowledgements}

The authors are deeply grateful to Antonio Segatti for the fruitful discussions and his encouragement during the preparation of this paper. The financial support of the MIURPRIN Grant 2008ZKHAHN "Phase transitions, hysteresis and multiscaling" and of the IMATI of CNR in Pavia is gratefully acknowledged.

\section{References}

[1] S.M. Allen, J.W. Cahn, A microscopic theory for the antiphase boundary motion and its application to antiphase domain coarsening, Acta Metallurgica, 27, 1085-1095, (1979).

[2] V. Barbu, Nonlinear Semigroups and Differential Equations in Banach Spaces, Noordhoff International Publishing, Leyden, 1976.

[3] V. Barbu, P. Colli, G. Gilardi, M. Grasselli, Existence, uniqueness, and longtime behavior for a nonlinear Volterra integrodifferential equation, Differential Integral Equations, 13, 1233-1262, (2000).

[4] H. Brézis, Opérateurs Maximaux Monotones et Semi-Groupes de Contractions dans les Espaces de Hilbert, Number 5 in North. Holland Math. Studies, North-Holland, Amsterdam, 1973.

[5] F. Brezzi, G. Gilardi, Chapters 1-3 in Finite Element Handbook, Edited by H. Kardestuncer and D.H. Norrie, McGraw-Hill Book Co., New York, 1987.

[6] M. Brokate, J. Sprekels, Hysteresis and Phase Transitions, Springer, New York, 1996.

[7] G. Caginalp, An analysis of a phase field model of a free boundary, Arch. Rational Mech. Anal., 92, 205-245, (1986).

[8] J.W. Cahn, J.E. Hilliard, Free energy of a nonuniform system I. Interfacial free energy, J. Chem. Phys., 2, 258-267, (1958).

[9] L. Calatroni, On the Allen-Cahn equation with singular potentials and dynamic boundary conditions, Master Thesis, Università degli studi di Pavia, a.a. 2010/2011.

[10] R. Chill, E. Fašangová, J. Prüss, Convergence to steady state of solutions of the CahnHilliard and Caginalp equations with dynamic boundary conditions, Math. Nachr., 13, 1448-1462, (2006).

[11] C.M. Elliott, S. Zheng, Global existence and stability of solutions to the phase-field equations, in Free Boundary Problems, Internat. Ser. Numer. Math., 95, pp. 46-58, Birkhäuser, Basel, 1990.

[12] X. Feng and A. Prohl., Numerical analysis of the Allen-Cahn equation and approximation for mean curvature, Numer. Math., 94, 33-65, (2003). 
[13] C.G. Gal, M. Grasselli, The non-isothermal Allen-Cahn equation with dynamic boundary conditions, Discrete Contin. Dyn. Syst., 22, 1009-1040, (2008).

[14] C.G. Gal, M. Grasselli, On the asymptotic behavior of the Caginalp system with dynamic boundary conditions, Commun. Pure Appl. Anal., 8, 689-710, (2009).

[15] G. Gilardi, A. Miranville, G. Schimperna, On the Cahn-Hilliard equation with irregular potentials and dynamic boundary equations, Commun. Pure Appl. Anal., 8, 881-912, (2009).

[16] M. Grasselli, V. Pata, Asymptotic behavior of a parabolic-hyperbolic system, Commun. Pure Appl. Anal., 3, 849-881, (2004).

[17] M. Grasselli, H. Petzeltovà, G. Schimperna, Long time behavior of solutions to the Caginalp system with singular potential, Z. Anal. Anwend., 25, 51-72, (2006).

[18] J.L. Lions, E. Magenes, Problèmes aux Limites non Homogènes et Applications, Vol. I, Dunod, Paris, 1968.

[19] A. Miranville, S. Zelik, Robust exponential attractors for singuarly perturbed phasefield type equations, Electron. J. Differential Equations, 63, 1-28, (2002).

[20] G. Schimperna, Abstract approach to evolution equations of phase-field type and applications, J. Differential Equations, 164, 395-430, (2000).

[21] J. Simon, Compact sets in the space $L^{p}(0, T ; B)$, Ann. Mat. Pura Appl., 146, 65-96, (1987).

[22] J. Sprekels, H. Wu, A note on parabolic equation with nonlinear dynamical boundary condition, Nonlinear Anal., 72, 3028-3048, (2010).

[23] A.A. Wheeler, W.J. Boettinger, G.B. McFadden, Phase-field model for isothermal phase transitions in binary alloys, Phys. Rev. A, 45, 7424-7439, (1992). 